\title{
Metalinguistic awareness in L2 vocabulary acquisition: Which factors influence learners' motivations of form-meaning connections?
}

\author{
Sarah Candry, Julie Deconinck \& June Eyckmans
}

Research has shown that prompting learners to elaborate on the appropriateness of form-meaning links can be an efficient vocabulary learning exercise (Deconinck, Boers \& Eyckmans, 2017). In this paper we wish to shed more light on the mental processes that occur during this specific elaborative task by investigating the influence of individual learner variables pertaining to prior linguistic knowledge and a number of word-specific features. To this end fifty Dutch-speaking EFL learners rated the congruency they perceived between the form and meaning of 24 English words on a 6point Likert scale. The motivation of their scores was elicited by means of a think-aloud protocol, the transcriptions of which were analysed with regard to the type of elaborations made. Vocabulary size tests and a language background questionnaire provided us with additional information about the learners. We identified five types of elaborations: cross-lexical associations, sound-symbolic associations, word-form comparisons, morphological associations, and idiosyncratic associations. The data also reveal that the individual learner variables and word-specific features examined in the present study have an influence on the number of elaborations made by the learners. Pedagogical implications and suggestions for further research are discussed.

Keywords: L2 vocabulary learning, Cognitive Linguistics, Metalinguistic awareness, SLA, vocabulary teaching 


\section{Introduction}

Cognitive linguists champion the idea that, in contrast to what de Saussure (1959) claimed, the relationship between form and meaning in language is not entirely arbitrary. In this light, they strive to find motivation in language, which entails that a retrospective explanation can be found as to why a certain word has a particular meaning for example (Radden \& Panther, 2004). From a vocabulary learning perspective, once an L2 learner finds a word to be linguistically motivated, it should be easier for said learner to remember this word. This is explained by the fact that considering the connection between a particular word form and its meaning creates a memory trace which facilitates recall of this word (Deconinck, Boers, \& Eyckmans, 2010, 2017). The notion of linguistic motivation thus provides learners with an opportunity to develop a new strategy of thinking about why word form and word meaning fit, and consequently employ this technique as a mnemonic method for remembering new L2 words generally (Beréndi, Csábi, \& Kövecses, 2008; Boers \& Lindstromberg, 2008a; Deconinck et al., 2017).

The present paper investigates a form-meaning-fit motivation task, which is a task that encourages L2 learners to consider 'the form-meaning fit' of a new word, i.e. to consider how well the form of the word matches its meaning (Deconinck et al., 2010; Deconinck, Boers, \& Eyckmans, 2014; Deconinck et al., 2017). This type of activity stimulates learners to produce associations, or rather elaborations between form and meaning. Deconinck et al. (2014) demonstrated that learners made four different types of form-meaning associations during the form-meaning-fit motivation task. We aim to determine whether the same associations can be discerned when employing the same method, but working with a different set of words and a different group of L2 learners. Analysis of these elaborations may reveal which formal features of L2 vocabulary are salient to the learners, and it can show the kind of meaningmaking learners are capable of during deliberate word learning in general. From a 
pedagogical point of view, this information is highly valuable, as it can help teachers in the classroom to render explicit the implicit associations learners make upon first seeing new L2 words. We will also explore the influence of individual learner factors on the elaboration process, for it is likely that not all learners are susceptible to seeing form-meaning connections. In particular, we will be investigating the effect of the learners' prior linguistic knowledge - that is, their L1, L2 and L3 knowledge - on their ability to make form-meaning associations. In this respect, we will also look at the influence of the learners' L2 vocabulary size and the number of languages they know. Furthermore, the extent to which learners elaborate upon a new word does not merely depend on their ability to do so; it may also be contingent on the word itself. Therefore, we will attempt to ascertain whether certain wordspecific features have an influence on the form-meaning-fit motivation process.

\section{Literature review}

Elaborating on new L2 words is an essential part of the vocabulary learning process. As pointed out by many scholars, learners should first and foremost notice an unknown word (Schmidt, 1990), a process which will be easier if the word form is more salient (e.g.

Dekeyser, 1998; Doughty, 2003; Gass \& Selinker, 2001). Once the word has been noticed, however, the chances of it being committed to memory are enhanced if the learner actively elaborates upon - or put differently, cognitively engages with - this word (e.g. Barcroft, 2002, 2003; Hulstijn, 2001). The more the learner engages with this word, the stronger its memory trace will be in the learner's mind (Laufer \& Hulstijn, 2001; Schmitt, 2008).

Considering whether the form-meaning connection of a word or multiword unit is motivated is one way of elaborating on new L2 vocabulary. Research has indicated that such an exercise facilitates L2 vocabulary learning. Boers, Eyckmans, and Stengers (2006), for instance, found that when learners understand how the lexical make-up of multiword units matches their meaning, it is easier for the learners to recall these multiword units. Deconinck 
et al. $(2014,2017)$ conducted a think-aloud protocol during which the learners were asked to rate how well the form of a new word fits its meaning, and to motivate why they gave a particular rating, thereby encouraging them to engage with both form and meaning. They found that the more a learner elaborated on an unknown L2 word, the better form recall was. Furthermore, they established that learners make four different types of form-meaning associations: cross-lexical associations, word-form comparisons, sound-symbolic associations, and idiosyncratic associations. The first two types of associations rely on the L1, L2 and/or L3 vocabulary that a learner has previously acquired. The data suggest that L2 learners capitalize greatly on their prior linguistic knowledge while being encouraged to make form-meaning elaborations.

Larsen-Freeman and Long (1991) and Bowden, Sanz and Stafford (2005) regard prior experience as an individual learner variable which clearly influences the learner's L2 learning process. It appears that during L2 vocabulary acquisition in general, learners - either consciously or unconsciously - exploit the linguistic knowledge they have already acquired from their L1 or L2, as posited by Hall's (2002) Parasitic Word Learning Hypothesis. The Parasitic Word Learning Hypothesis contends that when learners see a new L2 word, they will unconsciously try to capitalize on known L1 or L2 vocabulary; known words which display a certain amount of phonological or orthographic overlap with the new word will automatically be summoned up during processing. According to Hall (2002, p. 71), L2 vocabulary learning can be regarded 'as a problem of pattern-matching and assimilation with current lexical knowledge, at least at the onset of the word learning process'. When performing the formmeaning-fit motivation task, which in Deconinck et al.'s $(2010,2017)$ studies was deployed for initial L2 vocabulary learning, learners indeed seem to assimilate the new L2 vocabulary to their previously acquired lexical knowledge. 
By connecting L2 words to previously known vocabulary in the L1, L2 or L3, the learner seemingly constructs pathways that help to retrieve the target vocabulary. The outcome of Hall's experimental study provides support for this hypothesis. Spanish-speaking learners of English were given a number of English nonwords which were either pseudocognates - that is, nonwords which overlap in form with real L1 words - or noncognates. They were instructed to rate their familiarity with these words and to write down an L1 word which in their opinion was nearest in meaning to the given word. The data show that the pseudocognates felt more familiar to the learners, even though the learners had never seen them before. In addition, there appeared to be more consistency among the L1 translations provided by the participants for the pseudocognates than for the noncognates. Hall concluded that these results were due to the formal similarity between the pseudocognates and real L1 words. In the same light, Pierson (1989) advocates the meaningful learning approach: learners should be supplied with activities which raise their awareness of the relationships between new L2 words and other known words in their vocabularies since this will prompt them to create pathways for retrieval. Once learners are capable of forming such links, Pierson claims the learning burden of the new L2 vocabulary will be reduced.

If a learner's L2 proficiency can be considered to be an element of prior linguistic knowledge, research has indeed shown that, as a learner becomes more proficient in an L2 or L3, his/her level of metalinguistic awareness will increase (Jessner, 1999; Ringbom, 1987; Roehr, 2008). Jessner (2006, p. 42) defines metalinguistic awareness as 'the ability to focus attention on language as an object in itself or to think abstractly about language and, consequently, to play with or manipulate language'. Evaluating the link between the form and the meaning of an L2 word is undoubtedly an act which requires a certain amount of metalinguistic awareness. 
Metalinguistic awareness is argued to be only one component of linguistic awareness, however (Jessner, 2006). Another dimension is crosslinguistic awareness, which entails that the learner recognizes the similarities between different linguistic systems. In the process of becoming more proficient in an L2 or L3, the learner should become more aware of these similarities with their L1 and begin to actively seek them out. To help develop a learner's ability to think crosslinguistically, teachers should explicitly point out these resemblances (Jessner, 1999, 2006). Consequently, an important role is reserved for the language teacher in this process. Since learners' processing capacities tend to be too limited to focus on both the meaning and the form of new vocabulary during online processing, they are more likely to pay attention to meaning when encountering a new word (VanPatten, 1990). In addition, word form is limited in terms of the opportunities it gives learners to engage with it (Barcroft, 2002; Deconinck et al., 2014, 2017). It is then the teacher's task to explicitly draw the learners' attention to the form of the new L2 vocabulary (Boers \& Lindstromberg, 2008b: LauferDvorkin, 2006), for instance by pointing out how a certain word or multiword unit is motivated. To give but one example: the Dutch word 'hoed' looks similar to its English equivalent 'hat'. The teacher should explain how both form and meaning are similar and, thus, render the word motivated to the learner.

A second aspect of prior linguistic knowledge is the number of languages a learner has acquired previously, which can also influence any subsequent language learning processes. Research has demonstrated that bilingual learners acquiring a third language appear to have an edge over monolingual learners when attempting to acquire the same language (Cenoz \& Valencia, 1994; Sanz, 2000). The more languages a learner knows, the better this learner will be able to acquire an additional one (Jarvis, 2015). With each new language learners acquire, their level of metalinguistic awareness will also increase (Jessner, 2006). As their level of metalinguistic awareness grows, learners will be better able to exploit prior linguistic 
knowledge, both from the L1 and other acquired languages (Jarvis 2015). The benefit for bilingual or multilingual learners will be even stronger if the target language is typologically related to one of the languages they already know (Jarvis, 2015).

\section{Research questions}

Deconinck et al. (2014) discerned four types of form-meaning associations in their data: cross-lexical associations, sound-symbolic associations, word-form comparisons, and idiosyncratic associations. The form-meaning associations made might, however, be contingent on the individual language learner making the association, or on the properties of the word on which the association is based. The aim of the present paper is therefore to provide an answer to the following research questions:

(1) Which types of elaborations do learners make when asked whether the form of a particular L2 word fits its meaning?

Our aim is to corroborate Deconinck et al.'s (2014) findings by determining whether the same types of associations can be found when performing the form-meaning association exercise with a different set of words and a different group of language learners.

(2) Do the number of known languages and L2 vocabulary size influence the number of elaborations made by the learners?

We will investigate the influence of two individual learner factors pertaining to prior linguistic knowledge on the associations made. Firstly, we will investigate the effect of L2 vocabulary size. Studies have demonstrated that learners' L2 vocabulary size is correlated with their L2 proficiency in general. Hence, the vocabulary size tests provide us with an indication of the learners' L2 proficiency. We expect that learners with a larger L2 vocabulary size and learners mastering a larger number of languages will make more form-meaning associations, since they ought to possess an increased level of metalinguistic awareness 
(Jessner, 1999, 2006; Ringbom, 1987; Roehr, 2008), which should assist them during the process of form-meaning elaboration. They simply should have more linguistic knowledge to refer to. In this light, we also aim to determine whether typological relatedness between the languages known has a bearing on the type of associations made.

(3) Do word-specific features influence the learners' perception of the form-meaning fit?

First of all, we expect that the learners will make more cross-lexical associations when prompted by target words that have more English orthographic neighbours, since learners simply have more opportunities for making cross-lexical associations when there is a high number of words similar to the target word. We also predict that longer words will trigger more elaborations, since longer words contain more elements on which learners can base their associations. In addition, we expect pseudowords created by changing one letter in an existing high-frequency English word to evoke more cross-lexical associations than low-frequency English words, simply because the former better resemble known English words than the latter.

\section{Methodology}

\section{Participants}

Fifty adult EFL learners (13 male, 37 female) participated in the experiment. 49 participants had Dutch as their L1 and one participant indicated that Russian was his mother tongue. However, this participant had been living in a Dutch-speaking country for the most part of his life and remarked that he was more proficient in Dutch than in his mother tongue. The experiment was conducted at a university in Flanders, Belgium. The participants answered a call for volunteers and were awarded 40 euros for their participation. The participants' average age was 23 . The average age at which they first came into contact with English media 
was 11 and the average age at which they started acquiring English was 12, which is the age at which English instruction typically starts in Flemish classrooms. All of the participants had received formal English instruction during secondary school, with a typical length of 6 years. All participants were multilingual, with 12 of the learners speaking two languages, 22 speaking three languages, ten speaking four languages, four speaking five languages, one speaking seven languages and one learner speaking nine languages. These data are based on self-reports by the participants.

\section{Target words}

The learners were instructed to rate the form-meaning fit of 24 English words, half of which were low-frequency words and half of which were pseudowords ${ }^{i}$ (see appendix). All words were between five and seven letters long. The pseudowords were created by changing one letter of a real English word, so that they were orthographically and phonologically legal in the target language. In the rating task, all words were presented with their Dutch equivalents (i.e. their meanings) to allow the learners to rate the form-meaning fit of these words adequately. The number of orthographic neighbours of the target vocabulary was determined with the MCWord database (Medler \& Binder, 2005).

\section{Procedure}

The form-meaning-fit motivation task was added to a word learning procedure which was administered in the framework of another study (Elgort, Candry, Boutorwick, Eyckmans, \& Brysbaert, 2016). The complete procedure was carried out over the course of two days by the first author of this paper. Prior to the word learning procedure, a language background questionnaire was administered via email. Then, the participants learned the 24 words contextually: they were shown three contexts which contained the target word and were subsequently given the definition of each word. Next, the participants conducted a meaning 
recall test, and one day later, a gap-fill task tested their knowledge of word form. Then, two English vocabulary size tests were administered: the LexTale test (Lemhöfer \& Broersma, 2012), which measured receptive vocabulary size, and the Productive Vocabulary Levels Test (Laufer \& Nation, 1999) at the 2K, 3K, and 5K level, which gauged the participants' productive vocabulary size.

Next, the form-meaning-fit motivation task was administered. The learners received a list with the 24 English words and their Dutch equivalents. They were instructed to rate how well the form of each word matched its meaning on a 6-point Likert-scale $(1=$ completely disagree and $6=$ completely agree). Immediately after the ratings were given, the learners were asked to motivate why they had given these particular ratings during a think-aloud protocol conducted in Dutch. The experimenter only inquired after the words that they had allotted a score of one, two, five or six, since it was expected that the participants would have stronger opinions about these words than about those which they had given a fairly neutral score of three or four. If the participants appeared to experience difficulties in explaining why the form of a certain word did or did not fit its meaning, the experimenter attempted to elicit additional elaborations by asking questions such as 'Does this word remind you of something?'.

\section{Coding}

The transcriptions of the think-aloud protocols were first analysed by means of the coding manual employed by Deconinck et al. (2014), which contained four elaboration types: crosslexical associations, sound-symbolic associations, word-form comparisons, and idiosyncratic associations. After the first analysis, however, it appeared that the transcriptions contained a number of elaborations which could not be classified under any of the four categories. The coding manual was then adjusted, and the transcriptions were coded a second time by the same rater, as well as by a second rater. The two raters' codings were compared, and it was 
established that the inter-rater agreement was $80 \%$. The two raters then discussed the diverging codings and came to a consensus.

\section{Analysis}

To investigate which factors predict the number of elaborations made, a poisson regression was conducted by means of the glm function in R (R Core Team, 2015). The best model fit was selected on the basis of the AICc value of the model, which was determined with the AICc function in the MuMIn package (Barton, 2007).

\section{Results}

\section{Types of Associations}

The participants were only invited to reflect on the words they had given a form-meaning-fit rating of $1,2,5$, or 6 . As a result, 734 of the 1200 rated items were discussed during the thinkaloud procedure, which amounts to $61 \%$. Of these, 98 did not prompt any elaborations, 454 items prompted one elaboration, 144 items prompted two elaborations, 29 items prompted three elaborations, and only nine items prompted four associations. In total, 870 elaborations were observed. The learners' elaborations were classified into five categories: cross-lexical associations (CLA), sound-symbolic associations (SSA), word-form comparisons (WFC), morphological associations (MA), and idiosyncratic associations (IA). The categories are described below, and their frequencies are displayed in Table 1.

[Table 1 near here]

We will demonstrate the types of elaborations made by means of examples from the thinkaloud protocols. The form-meaning-fit rating given for these particular cases is included. Since the think-aloud protocols were conducted in Dutch, we have provided an English translation of the participants' reflections on the form-meaning fit of the target vocabulary. 


\section{Cross-Lexical Associations}

A cross-lexical association (CLA) entails that the target L2 word triggered another word present in the L1, L2 or L3 lexicon of the learner because of the resemblance in word form. Hence, the elaboration is based on the similarity in word form between the target L2 word and the triggered word. Cross-lexical associations with the L2 were the most frequently occurring type. The example below demonstrates how a CLA with an L2 word can induce a process of meaning-making in this type of exercise.

\section{$\underline{\text { busser - afruimer (table cleaner) - Form-meaning-fit rating: } 6}$}

Participant 4: yeah because yeah also bus it stops at every stop, but you can't expect a superb service and that's the same as a busser does, that person has stops and clears all the tables but he doesn't really interact with the customers

\section{Word-Form Comparisons}

A word-form comparison implies that the learner compared the form of the target word with the form of another word - either in the L1 or L2 - that has the same meaning. This entails that the learner first thought of a word with the same meaning as the target word and then compared the form of this prompted word with the target word. Hence, this type of elaboration is based on the resemblance in meaning between the L2 word and the elicited word. Learners either made word-form comparisons with a Dutch word (WFC L1) or with an English word that had the same meaning as the target word (WFC L2), as demonstrated in the example below.

ladle - soeplepel (spoon used for serving soup) - Form-meaning-fit rating: 2 
Participant 44: yeah I also don't think that's, you know, you would think that that is also spoon or something like that like with us. A lepel (spoon) or a soeplepel (soup spoon) that that also looks alike while it is the same object in the end \# that's why I think it's weird that that's suddenly a completely different word

So in this instance the participant is reflecting on the fact that ladle and spoon do not look alike despite their semantic link, whereas their perceived Dutch counterparts, i.e. lepel and soeplepel, do look alike.

\section{Morphological associations}

Learners also made use of morphological associations, which are associations based on the English morphological knowledge they have previously acquired. Morphological associations can entail that the learner breaks the word up into smaller parts, as in the following example:

\section{egress - nooduitgang (emergency exit) - Form-meaning-fit rating: 5}

Participant 7: e- is always exit out and and -gress comes from a verb which means to go

Another type of morphological association discerned in the data involved associating the form of a word with a specific word class or a specific number (i.e. singular or plural), as demonstrated in the following example:

$\underline{\text { recresh - luchtbellen (air bubbles) - Form-meaning-fit rating: } 1}$

Participant 19: first of all it says luchtbellen (air bubbles) and recresh does not seem an $\langle$ uhm $>$ does not sound plural to me so that is $\langle u h m>$ already one thing and erm recresh I don't see that as a $<$ uhm $>$ a noun 


\section{Sound-symbolic Associations}

A sound-symbolic association implies that the learner attributed sound-symbolic features to the word. This means that a resemblance was perceived between the sound or shape of a word and the perceptual properties of its referent. The category can be further subdivided into three types of sound-symbolic associations. Learners can find sound-symbolism in the entire word or sizeable word part, in individual sounds, or in the letters or spelling of the word as a whole, although this last type of elaboration was very rare. The example below demonstrates how a learner found sound-symbolism in an entire word.

\section{dollop - klodder, kwak (lump) - Form-meaning-fit rating: 5}

Participant 10: a dollop also has something \# a sound of something that \# a a blob of something that is slapped onto something or yes

\section{Idiosyncratic associations}

This category comprises form-meaning elaborations that were spontaneously produced by the learner but in which no pattern could be discerned, as is demonstrated in the following example:

clabber - karnemelk (yogurt-like substance) - Form-meaning-fit rating: 6

Participant 16: yeah at first I thought it was some sort of English or Irish dish clabber

Besides these types of elaborations, learners made other meaningful elaborations, but ones that did not contemplate the form-meaning link itself. For instance, participants often made utterances such as 'this is a nice word' or 'this is a strange word', but did not give any more explanation as to why they found this. This type of elaboration could be termed 
phonological attraction and made up $14 \%$ of the total number of meaningful utterances, but they will not be included in the analysis since they are not form-meaning associations.

We also investigated whether the number of elaborations made can be predicted by certain individual learner variables and word-specific features. The results of the analysis are given in Table 2.

[Table 2 near here]

\section{Individual learner factors}

The effect of two individual learner variables was investigated: L2 vocabulary size (both receptive and productive) and number of known languages. Firstly, we report the results of the two English vocabulary size tests. The learners' average test scores on the Productive Vocabulary Size Test (Laufer \& Nation, 1999) were 15.8 (=88\%) at the 2000 word frequency level, $12.8(71 \%)$ at the 3000 word frequency level, and $9(=50 \%)$ at the 5000 word frequency level. Their average score on the LexTALE test (Lemhöfer \& Broersma, 2012), which measures receptive vocabulary size, was $74.6 \%$. Apparently, receptive L2 vocabulary size has a predictive value for the number of associations made by the learners, as is demonstrated by the second line of Table 2. The larger a learner's receptive L2 vocabulary size, the more associations this learner makes. This effect is also observed in the analysis of cross-lexical associations with the $\mathrm{L} 2\left(\right.$ Estimate $\left.=0.018, \mathrm{SE}=0.006, \mathrm{z}=2.980, \mathrm{p}=0.003, \mathrm{R}^{2}=0.137\right)$ and word-form comparisons with the L2 (Estimate $=0.03, \mathrm{SE}=0.015, \mathrm{z}=2.014, \mathrm{p}=0.044, \mathrm{R}^{2}=$ 0.023). Productive L2 vocabulary size significantly predicts the number of cross-lexical associations made with the L2 (Estimate $=0.054, \mathrm{SE}=0.027, \mathrm{z}=1.976, \mathrm{p}=0.048, \mathrm{R}^{2}=$ 0.137), but does not predict the total number of associations.

Number of known languages does not predict the total number of associations, as can be seen on the third line of Table 2. The number of these known languages which is 
typologically related to English also does not affect the number of elaborations made by the learners.

\section{Word-specific features}

As can be seen on the fourth line of Table 2, word length has a significant influence on the number of associations made: The longer the word was, the more associations it triggered. Number of orthographic neighbours does not have a significant influence on the total number of associations made, but words with more orthographic neighbours did trigger more crosslexical associations with the L2 (Estimate $=0.335, \mathrm{SE}=0.075, \mathrm{z}=4.494, \mathrm{p}<0.001, \mathrm{R}^{2}=$ 0.137). Word type, i.e. whether the word was a low-frequency word or a pseudoword, does not influence the total number of elaborations made, although pseudowords do yield a significantly higher number of cross-lexical associations with the L2 than low-frequency words $\left(\right.$ Estimate $\left.=1.165, \mathrm{SE}=0.075, \mathrm{z}=7.127, \mathrm{p}<0.001, \mathrm{R}^{2}=0.137\right)$.

\section{Discussion}

The aim of the present study was to corroborate the already existing form-meaning elaboration categories which learners make during the form-meaning-fit motivation task, as established by Deconinck et al. (2014), in order to determine whether this technique triggers the same patterns under different circumstances. The categories we discerned were indeed largely the same as those distinguished by Deconinck et al. (2014), which demonstrates that the learners unconsciously produce the same types of connections based on their prior knowledge (although it must be noted that the frequency with which the different types of elaborations occur vary due to individual learner variables and word-specific features). Therefore, we can assume that language learners in general possess a capacity for elaborating on the form-meaning fit of L2 vocabulary. However, we were not only able to verify the occurrence of the existing form-meaning elaboration types, but were also able to further 
expand on these types by establishing an additional form-meaning elaboration category, namely morphological associations. We categorized these elaborations into a separate category since learners who employ this type of elaboration demonstrate that they possess morphological awareness, which can also be regarded as a type of metalinguistic awareness (Nagy, 2007). Studies have indicated that learners who apply morphological analysis as a vocabulary learning strategy, and as such possess metalinguistic awareness, perform better during the L1 and L2 vocabulary learning process (Freyd \& Baron, 1982; Morin, 2003; Nagy \& Anderson, 1984; Tyler \& Nagy, 1989; White, Power, \& White, 1989).

The distribution of the categories in the present study does differ from Deconinck et al. (2014). Cross-lexical associations were again the most frequent type of elaborations, followed by word-form comparisons as the second most frequent category. Morphological associations came third, followed by sound-symbolic associations. Idiosyncratic associations were the least frequent type of elaboration. Deconinck et al. (2014), on the other hand, found that sound-symbolic associations were the second most frequent category and that word-form comparisons came third. This could be because the target words used in the present study contained fewer properties which induced these sound-symbolic associations than the target words used in Deconinck et al. (2014).

In this study, the form-meaning-fit motivation task followed a learning procedure in the framework of another study (Elgort et al., 2016) during which the target items had been learned by the participants. However, not all words had been acquired by the learners after this procedure. As such, the target items discussed during the form-meaning-fit motivation task were a mixture of both acquired and not acquired words. We have verified whether knowledge of the word influenced the number of elaborations a learner made on this word, and found that a learner's ability to elaborate on a particular word was not influenced by whether or not this word was known. Therefore, it appears that the form-meaning-fit 
motivation task can be applied during both initial word learning and at a more advanced stage of word learning. Once learners are familiar with a word, they can still apply the formmeaning-fit motivation task to integrate the word more firmly in their mental lexicon.

A crucial factor determining a learner's ability to elaborate on the form-meaning fit seems to be the extent of metalinguistic awareness this learner possesses. In the present study, we attempted to determine whether individual learner factors which are believed to influence the amount of metalinguistic awareness a learner possesses also affect this learner's ability to elaborate on the form-meaning fit of a word. The first variable we investigated was L2 vocabulary size. The results confirm our initial hypothesis that increased L2 vocabulary size, both receptive and productive, would have a positive effect on the number of elaborations made. The number of cross-lexical associations with the L2 and word-form comparisons with the L2 in particular increased as the learners' L2 vocabulary size increased. Naturally, it should be easier for learners to associate an L2 word with other L2 vocabulary if they have more words in their mental lexicons to make reference to. This finding is also in keeping with the idea that increased proficiency in the L2 results in a higher level of metalinguistic awareness (Jessner, 1999; Ringbom, 1987; Roehr, 2008), and as such also in a heightened ability to elaborate on L2 vocabulary.

The number of languages known by the learner, on the other hand, did not have an influence on this learner's ability to elaborate on the target vocabulary in the context of our study. This could be due to the fact that all participants were either bilingual or multilingual. According to Jessner (2008, p. 277), the metalinguistic awareness developed by monolinguals 'cannot be compared in both degree and quality to awareness as developed in bi- and multilingual users'. As such, all participants have a more developed level of metalinguistic awareness than learners who only speak one language. Perhaps an effect would have been perceived if we had compared multilingual learners with monolinguals who are at an early 
stage of L2 learning. In the context of a multilingual country such as Belgium, such monolingual participants are hard to find, especially within this age range. Moreover, all learners were native Dutch speakers and thus mastered at least one typologically related language. During the form-meaning-fit motivation exercise, only fifteen elaborations were made with an L3. Of these, fourteen were made with a word from a Romance language. Only one CLA L3 was made with a typologically related language, namely German. In general, learners resorted to the L2 and their typologically related L1 to make cross-lexical associations and word-form comparisons with the target vocabulary. One explanation for this finding may be that because the think-aloud procedures were conducted in Dutch with English words, the associations that were neither Dutch nor English were simply not expressed. After all, even if think alouds are considered introspective, they occasion a dialogue between participant and experimenter, which turns it into a social and collaborative task (cf. Dörnyei, 2007; Kussmaul \& Tirkkonen-Condit, 1995). If multilinguals assumed their interlocutor did not have the same linguistic background as them, then a social desirability bias might have prevented them from voicing their own personal associations.

During the procedure, we also questioned the learners on their use of three vocabulary learning strategies that all involve harnessing prior linguistic knowledge: performing a word analysis (i.e. breaking the word down into smaller parts); comparing the form of the L2 word with another word which has a similar form (e.g. the English word 'book' and the Dutch word 'boek'); and/or comparing the form of the L2 word with another word which has the same meaning (e.g. the English word 'coat' and the Dutch word 'jas'). The most frequently reported vocabulary learning strategy was connecting the form of the new word with a word that resembles it. Of the fifty participants, 36 indicated they spontaneously use this strategy. Comparing the new word with another word that carries the same meaning came second, with 25 participants signalling that they use this strategy. This strategy is more or less on par with 
word analysis, of which 24 participants state that they use this strategy. The three techniques correspond to three of the types of elaborations learners made during the task, namely morphological analyses, cross-lexical associations, and word-form comparisons. We expected that if learners indicate that they make use of such vocabulary learning strategies, it would imply that they spontaneously capitalize on prior linguistic knowledge during the L2 vocabulary learning process, and that they would be more capable of thinking about new vocabulary metalinguistically than those learners who did not attest to instinctively applying these vocabulary learning strategies. However, analysis demonstrated that this element did not predict the number of elaborations made by the learners. We can conclude that, at least in the present design, learners who indicate that they spontaneously make use of prior linguistic knowledge during vocabulary learning do not seem to make more form-meaning elaborations than those learners who have not yet developed the use of these vocabulary learning strategies, or at least, do not claim they have. Therefore, in our study the form-meaning-fit motivation task induced the same amount of form-meaning elaborations among all participants, regardless of whether they claimed to be familiar with certain types of elaborations prior to conducting the exercise for the first time.

With regard to word-specific features, we established that words with more orthographic neighbours elicited a significantly higher number of cross-lexical associations with the L2. Naturally, it should be easier for a learner to associate an L2 word with other L2 vocabulary if there are more L2 words that resemble the target word. For the same reason, the English pseudowords learned in this study, which were created by changing one letter in an existing English word, induced more cross-lexical associations with the L2. Learners were reminded of the real English words on which the pseudowords were based and formed a connection between both words. This again demonstrates how, as Hall (2002) posits in the Parasitic Word Learning Hypothesis, L2 learners search for similarities between unknown 
vocabulary and vocabulary which is already part of their mental lexicon. Longer words elicited significantly more form-meaning associations, because longer words offer the learners more elements to exploit and may contain a larger number of salient features. As such, longer words provide the learner with more possibilities to elaborate on.

\section{Conclusion}

Deconinck et al. $(2014,2017)$ found that elaborating on the potential form-meaning-fit motivation of an unknown L2 word is a technique which results in increased word recall gains and therefore can be exploited in the language learning classroom. In addition, they established four types of resulting elaborations. Our study, which employed a different set of target items and a different group of language learners, authenticated these categories and established an additional category. As such, the study reaffirms that L2 learners possess an ability to elaborate on new L2 vocabulary. In the present study, we detected five types of associations: cross-lexical associations, sound-symbolic associations, word-form comparisons, morphological associations, and idiosyncratic associations. Future research should investigate whether teaching the learners which elaborations they can make before they carry out the form-meaning-fit motivation exercise leads to even higher learning gains than those noted in Deconinck et al. (2017).

With regard to the number of elaborations, it appeared that the larger the receptive L2 vocabulary size of the learner, the better this learner was capable of making these elaborations. Knowing more languages, however, did not appear to impact the number of elaborations a learner makes. Whether these languages were typologically related or not also did not play a part in the learners' performance on the form-meaning-fit motivation task. We observed that, in fact, all learners exploited the prior linguistic knowledge from their typologically related L1, but did not exploit the knowledge from other typologically related languages they mastered. Further research should carry out the exercise with L2 learners 
whose L1 is not typologically related to their L2. Such a study could uncover whether the learners exploit the linguistic knowledge they have acquired from languages that are typologically unrelated to the L2. With respect to word-specific features, we established that longer words induced more elaborations than the shorter items. In addition, words with more English orthographic neighbours and pseudowords induced more cross-lexical associations with other L2 words.

\section{Pedagogical implications}

Since we generally established the same types of form-meaning elaborations as Deconinck et al. (2014), we can assert that language learners have an ability to motivate the form-meaning connection of an L2 word. Language teachers can tap into this ability and employ the formmeaning-fit motivation method to promote acquisition of new L2 words. In order to train language learners to apply the method, it is important that they are aware of the different types of elaborations learners can make during such an activity. Pierson (1989) and Jessner (1999, 2006) already suggested that learners should be made aware of the similarities between new L2 vocabulary and other words present in their mental lexicon, be it in the L1, L2 or L3. It is important, though, that teachers also point to the possible dangers of simply assuming that new L2 words resembling other words in the learner's mental lexicon have a similar meaning. For instance, false friends, i.e. words from different languages which resemble each other but have diverging meanings, could steer the learners in the wrong direction. Hence, the formmeaning-fit motivation technique should never be used as a guessing technique. Instead, language teachers should always motivate learners to look up the meaning of an unknown word and only then elaborate on the form-meaning fit of this word. It stands to reason that the form-meaning fit of a word can only be properly assessed when the meaning part of the equation is correct. 
The results of this study revealed that the receptive L2 vocabulary size of the learners had an influence on the number of elaborations made. Language teachers should therefore take into account the level of L2 proficiency, and in particular the level of L2 vocabulary knowledge, of the group of learners they are teaching when they apply the form-meaning-fit motivation technique. Beginning L2 learners should be offered more guidance, since they are not as capable of making form-meaning elaborations as advanced L2 learners. They should be presented with additional examples and practice to learn how the technique works. Learners should also be informed about other possible types of elaboration which do not pertain to cross-linguistic similarity, such as sound-symbolic associations. When prompted, the learners in our treatment spontaneously employed these other types of elaborations too, so further awareness-raising through the form-meaning-fit technique could result in the increased use of these elaborations as a vocabulary learning strategy. We also established that the word length and orthographic neighbourhood of a word impacted on the number of elaborations a learner made. These findings with regard to word-specific features can aid language teachers in determining for which words use of the form-meaning-fit motivation method might be most efficient.

\section{Limitations}

The present study has some limitations. For one, requesting the learners to elaborate on a rather large number of items successively may not have constituted an ecologically valid learning environment. In reality, learners may employ the form-meaning technique when they encounter a new word in class or are deliberately studying new vocabulary. In this case, they will only go through the process of thinking about the form-meaning-fit motivation of a single word, rather than a series of words. This may have had an influence on the participants' ability or willingness to engage with the vocabulary during the think-aloud procedure. Secondly, the learners reported themselves which languages they mastered, but we cannot 
verify the truthfulness of these self-reports; we did not have unbiased information about the learners' proficiency in these languages. Thirdly, we exclusively focused on individual learner variables pertaining to prior linguistic knowledge. In a future research project, other individual learner factors, such as working memory capacity, cognitive style or learner style should be taken into account, since these might also exert a considerable influence on the efficiency of the form-meaning-fit motivation method (Roehr, 2008).

We investigated word-specific features which were relevant especially to three types of form-meaning elaborations: English orthographic neighbourhood and word type were expected to have an influence on the number of cross-lexical associations made with L2 words, and word length had an influence on the number of cross-lexical associations, wordform comparisons and morphological associations, since longer words provided the learners with more material on which they could base these elaborations. A future study should be directed at other word-specific features, such as the influence of sound-symbolic elements on the type of elaborations learners make. The sound-symbolic associations category was the fourth most frequent category in our study, whereas it was the second most frequent category in Deconinck et al.'s (2014) study. This might be due to the fact that the words in the present study contained fewer sound-symbolic features, so further research should bring clarity on this matter. Finally, we did not investigate the number of Dutch orthographic neighbours the target vocabulary had. If the words in this study had many orthographic neighbours in the learners' L1, this may have had an influence on the number of elaborations, and in particular on the number of cross-lexical associations with the L1. We determined the number of English orthographic neighbours by means of the MCWord database (Medler \& Binder, 2005). However, to our knowledge, no tool for determining the number of Dutch orthographic neighbours of English words had been developed yet. Consequently, we were not able to incorporate this factor in our study. This can also be the subject of future research. 


\section{References}

Barcroft, J. (2002). Semantic and Structural Elaboration in L2 Lexical Acquisition. Language Learning, 52(2), 323-363. doi:10.1111/0023-8333.00186

Barcroft, J. (2003). Effects of Questions about word meaning during Spanish L2 lexical acquisition. The Modern Language Journal, 87(4), 546-561. doi:10.1111/15404781.00207

Barton, K. (2007). Model selection and model averaging based on information criteria (AICc and alike). Retrieved from https://cran.r-project.org/web/packages/MuMIn/index.html, R package version 1.15.6.

Beréndi, M., Csábi, S., \& Kövecses, Z. (2008). Using conceptual metaphors and metonymies in vocabulary teaching. In F. Boers \& S. Lindstromberg (Eds.), Cognitive Linguistic Approaches to Teaching Vocabulary and Phraseology (pp. 65-100). Berlin: Mouton de Gruyter.

Boers, F., Eyckmans, J., \& Stengers, H. (2006). Motivating multiword units: Rationale, mnemonic benefits, and cognitive style variables. In S. H. Foster-Cohen, M. Medved Krajnovic, \& J. Mihaljevic Djigunovic (Eds.), EUROSLA Yearbook (Vol. 6, pp. 169190). Amsterdam: John Benjamins Publishing Company.

Boers, F., \& Lindstromberg, S. (2008a). How cognitive linguistics can foster effective vocabulary teaching. In F. Boers \& S. Lindstromberg (Eds.), Cognitive Linguistic Approaches to Teaching Vocabulary and Phraseology (pp. 1-64). Berlin: Mouton de Gruyter.

Boers, F., \& Lindstromberg, S. (2008b). Structural elaboration by the sound (and feel) of it. In Cognitive Linguistic Approaches to Teaching Vocabulary and Phraseology. (pp. 329353). (Cognitive Linguistic Approaches to Teaching Vocabulary and Phraseology). Mouton de Gruyter.

Bowden, H. W., Sanz, C., \& Stafford, C. A. (2005). Individual Differences: Age, Sex, Working Memory and Prior Knowledge. In C. Sanz (Ed.), Mind \& Context in Adult Second Language Acquisition. Washington, DC: Georgetown University Press.

Cenoz, J., \& Valencia, J. F. (1994). Additive trilingualism: Evidence from the Basque Country. Applied Psycholinguistics, 15(195-207).

de Saussure, F. (1959). Course in General Linguistics. New York: Philosophical Library.

Deconinck, J., Boers, F., \& Eyckmans, J. (2010). Helping learners engage with L2 words: The form-meaning fit. AILA Review, 23, 95-114. doi:10.1075/aila.23.06dec 
Deconinck, J., Boers, F., \& Eyckmans, J. (2014). Looking for form-meaning motivation in new L2 words: A think-aloud study among proficient learners of English. English Text Construction, 7(2), 249-280. doi:10.1075/etc.7.2.04dec

Deconinck, J., Boers, F., \& Eyckmans, J. (2017). 'Does the form of this word fit its meaning?' The effect of learner-generated mapping elaborations on L2 word recall. Language Teaching Research, 21, 31-53. doi:10.1177/1362168815614048

Dekeyser, R. (1998). Beyond focus on form: Cognitive perspectives on learning and practicing second language grammar. Focus on Form in Classroom Second Language Acquisition (pp. 42-63). Cambridge: Cambridge University Press.

Dörnyei, Z. (2007). Research Methods in Applied Linguistics. Oxford: Oxford University Press.

Doughty, C. (2003). Instructed SLA: Constraints, compensation, and enhancement. In C. Doughty \& M. Long (Eds.), Handbook of Second Language Acquisition (pp. 256-310). Oxford: Blackwell.

Elgort, I., Candry, S., Boutorwick, T. J., Eyckmans, J., \& Brysbaert, M. (2016). Contextual word learning with form-focused and meaning-focused elaboration: A comparative study. Applied Linguistics, advance online publication. doi:10.1093/applin/amw029

Freyd, P., \& Baron, J. (1982). Individual differences in acquisition of derivational morphology. Verbal Learning and Verbal Behavior, 21, 282-295.

Gass, S., \& Selinker, L. (2001). Second Language Acquisition: An Introductory Course. NJ: Erlbaum Associates.

Hall, C. J. (2002). The automatic cognate form assumption: Evidence for the parasitic model of vocabulary development. International Review of Applied Linguistics in Language Teaching, 40, 69-87. doi:10.1515/iral.2002.008

Hulstijn, J. (2001). Intentional and incidental second language vocabulary learning: a reappraisal of elaboration, rehearsal and automaticity. In P. Robinson (Ed.), Cognition and Second Language Instruction (3 ed., pp. 258-286). Cambridge: Cambridge University Press.

Jarvis, S. (2015). Influences of Previously Learned Languages on the Learning and Use of Additional Languages. In M. Juan-Garau \& J. Salazar-Noguera (Eds.), Content-based Language Learning in Multilingual Educational Environments (pp. 69-86). Berlin: Springer. 
Jessner, U. (1999). Metalinguistic Awareness in Multilinguals: Cognitive Aspects of Third Language Learning. Language Awareness, 8, 201-211. doi:10.1080/09658419908

Jessner, U. (2006). Linguistic awareness in multilinguals : English as a third language Edinburgh: Edinburgh University Press.

Jessner, U. (2008). A DST model of multilingualism and the role of metalinguistic awareness. The Modern Language Journal, 92(2), 270 - 283.

Kussmaul, P., \& Tirkkonen-Condit, S. (1995). Think-aloud protocol analysis in translation studies. TTR: Traduction, Terminologie, Rédaction, 8(1), 177-199. doi:10.7202/037201ar

Larsen-Freeman, D., \& Long, M. (1991). An introduction to second language acquisition research. London: Longman.

Laufer-Dvorkin, B. (2006). Comparing Focus on Form and Focus on FormS in SecondLanguage Vocabulary Learning. The Canadian Modern Language Review / La revue canadienne des langues vivantes, 63(1), 149-166. doi:10.1353/cml.2006.0047

Laufer, B., \& Hulstijn, J. (2001). Incidental Vocabulary Acquisition in a Second Language: The Construct of Task-Induced Involvement. Applied Linguistics, 22(1), 1-26. doi:10.1093/applin/22.1.1

Laufer, B., \& Nation, P. (1999). A vocabulary size test of controlled productive ability. Language Testing, 16(1), 33-51. doi:10.1177/026553229901600103

Lemhöfer, K., \& Broersma, M. (2012). Introducing LexTALE: a quick and valid Lexical Test for Advanced Learners of English. Behav Res Methods, 44(2), 325-343. doi:10.3758/s 13428-011-0146-0

Medler, D. A., \& Binder, J. R. (2005). MCWord: An On-Line Orthographic Database of the English Language (Medler \& Binder, 2005). Retrieved from www.neuro.mcw.edu/mcword/

Morin, R. (2003). Derivational Morphological Analysis as a Strategy for Vocabulary Acquisition in Spanish. The Modern Language Journal, 87(2), 202-221. doi:10.1111/1540-4781.00186

Nagy, W. (2007). Metalinguistic Awareness and the Vocabulary-Comprehension Connection. In R. Wagner, A. Muse, \& K. Tannenbaum (Eds.), Vocabulary acquisition: implications for reading comprehension. New York: The Guilford Press.

Nagy, W., \& Anderson, R. (1984). How many words are there in printed school English? Reading Research Quarterly, 19, 304-330. 
Pierson, H. (1989). Using etymology in the classroom. ELT Journal, 43(1), 57-63. doi:10.1093/elt/43.1.57

R Core Team (2015). $R$ : A language and environment for statistical computing. $R$ Foundation for Statistical Computing, Vienna, Austria. URL http://www.R-project.org/.

Radden, G., \& Panther, K.-U. (2004). Studies in Linguistic Motivation. Berlin: Mouton de Gruyter.

Ringbom, H. (1987). The Role of the First Language in Foreign Language Learning. Clevedon, PH: Multlilingual Matters.

Roehr, K. (2008). Linguistic and metalinguistic categories in second language learning. Cognitive Linguistics, 19(1). doi:10.1515/cog.2008.005

Sanz, C. (2000). Bilingual education enhances third language acquisition: Evidence from Catalonia. Applied Psycholinguistics, 21, 23-44.

Schmidt, R. W. (1990). The Role of Consciousness in Second Language Learning. Applied Linguistics, 11(2), 129-158. doi:10.1093/applin/11.2.129

Schmitt, N. (2008). Review Article: Instructed second language vocabulary learning. Language Teaching Research, 12(3), 329-363. doi:10.1177/1362168808089921

Tyler, A., \& Nagy, W. (1989). The acquisition of English derivational morphology. Journal of Memory and Language and Education, 28, 649-667.

VanPatten, B. (1990). Attending to content and form in the input: an experiment in consciousness. Studies in Second Language Acquisition, 12, 287-301. doi:10.1017/S027226310000917

White, T. G., Power, M. A., \& White, S. (1989). Morphological Analysis: Implications for teaching and understanding vocabulary growth. Reading Research Quarterly, 24(3), 283-304. 


\section{Appendix}

\section{Target vocabulary}

The tables show the twelve low-frequency words and the twelve pseudowords employed during the experiment. Short definitions have been added for the sake of the reader.

\begin{tabular}{|l|l|l|}
\hline Low-frequency words & Meaning & $\begin{array}{l}\text { Number of orthographic } \\
\text { neighbours }\end{array}$ \\
\hline egress & exit & 2 \\
\hline anvil & $\begin{array}{l}\text { heavy block on which metal is } \\
\text { shaped }\end{array}$ & 0 \\
\hline dibble & $\begin{array}{l}\text { gardening tool for making } \\
\text { holes }\end{array}$ & 2 \\
\hline gable & $\begin{array}{l}\text { triangular area of a house } \\
\text { beneath the roof }\end{array}$ & 4 \\
\hline pelmet & $\begin{array}{l}\text { border to hide the fittings of } \\
\text { curtains }\end{array}$ & 2 \\
\hline bodger & carpenter & 4 \\
\hline dollop & lump of soft food & 1 \\
\hline pepita & pumpkin seed & 0 \\
\hline busser & waiter's assistant & 3 \\
\hline griddle & $\begin{array}{l}\text { cooking surface with a heat } \\
\text { source underneath }\end{array}$ & 0 \\
\hline ladle & large spoon & 0 \\
\hline clabber & soured, fermented milk & 3 \\
\hline
\end{tabular}




\begin{tabular}{|l|l|l|}
\hline Pseudowords & Meaning & $\begin{array}{l}\text { Number of orthographic } \\
\text { neighbours }\end{array}$ \\
\hline parsage & woodcutting saw & 1 \\
\hline shottle & gravel & 1 \\
\hline spiler & plumber & 1 \\
\hline emback & wilderness hut & 2 \\
\hline banity & wall painting & 2 \\
\hline ferch & threshold & 2 \\
\hline troppy & gastronome & 1 \\
\hline tragger & colander & 1 \\
\hline gastle & pipette used to moisten food & 1 \\
\hline recresh & air bubbles & 1 \\
\hline capsale & appetizer & 1 \\
\hline bondit & fritter & \\
\hline
\end{tabular}




\section{Tables}

Table 1. Distribution and frequencies of the elaborations made by the participants

\begin{tabular}{|c|c|c|}
\hline Type of association & Number of occurrences & Percentage $(n=870)$ \\
\hline CLA & 399 & $45.8 \%$ \\
\hline CLA LI & 146 & $16.8 \%$ \\
\hline CLA L2 & 238 & $27.4 \%$ \\
\hline CLA L3 & 15 & $1.7 \%$ \\
\hline WFC & 168 & $19.3 \%$ \\
\hline WFC L1 & 129 & $14.8 \%$ \\
\hline WFC L2 & 39 & $4.5 \%$ \\
\hline MA & 148 & $17 \%$ \\
\hline SSA & 126 & $14.5 \%$ \\
\hline$S S W$ & 108 & $12.4 \%$ \\
\hline$S S S$ & 16 & $1.8 \%$ \\
\hline$S S L$ & 2 & $0.2 \%$ \\
\hline IA & 29 & $3.3 \%$ \\
\hline
\end{tabular}

Table 2. Influence of the independent variables on the total number of elaborations made by the learners, as demonstrated by the poisson regression

\begin{tabular}{lllll}
\hline & Estimate & Std. Error & z-value & p-value \\
\hline Intercept & -0.987 & 0.415 & -2.379 & $\mathrm{p}=0.0174$ \\
\hline Receptive L2 vocabulary size & 0.007 & 0.003 & 2.435 & $\mathrm{p}=0.0149$ \\
\hline Number of known languages & -0.026 & 0.029 & -0.905 & $\mathrm{p}=0.3654$ \\
\hline Word Length & 0.108 & 0.053 & 2.033 & $\mathrm{p}=0.0421$ \\
& & & & $\mathrm{R}^{2}=0.033$ \\
\hline
\end{tabular}

\footnotetext{
${ }^{\mathrm{i}}$ These words were first used by Elgort, Candry, Boutorwick, Eyckmans, \& Brysbaert (2016).
} 